Archives de sciences sociales des religions

136 | octobre - décembre 2006

Les Archives... cinquante ans après

\title{
Les Archives ...Cinquante ans... après
}

André Mary

\section{(2) OpenEdition}

Journals

Édition électronique

URL : http://journals.openedition.org/assr/3810

DOI : 10.4000/assr.3810

ISSN : $1777-5825$

Éditeur

Éditions de l'EHESS

Édition imprimée

Date de publication : 1 décembre 2006

Pagination : 9-24

ISBN : 2-7132-2124-2

ISSN : 0335-5985

Référence électronique

André Mary, « Les Archives ...Cinquante ans... après », Archives de sciences sociales des religions [En ligne], 136 | octobre - décembre 2006, mis en ligne le 04 avril 2007, consulté le 21 septembre 2020. URL : http://journals.openedition.org/assr/3810 ; DOI : https://doi.org/10.4000/assr.3810 


\section{André Mary \\ Les Archives \\ Cinquante ans... après}

La commémoration du cinquantenaire de la revue des Archives de Sciences Sociales des Religions aurait pu prendre bien des formes : hommage aux fondateurs ${ }^{1}$, retour sur l'histoire d'une entreprise singulière, numéro spécial sur l'état des lieux et les perspectives d'avenir des sciences sociales du religieux ${ }^{2}$. Chaque anniversaire de la revue a emprunté successivement ces diverses voies comme on le rappelle ci-dessous.

L'équipe de rédaction des Archives a fait le choix de s'engager, à cette occasion, dans un vaste projet de "patrimonialisation » de l'histoire de la revue et du groupe de recherche de sociologie des religions qui lui a donné naissance. Dans le complément du projet Persée qui a déjà permis la numérisation de l'ensemble des numéros de la revue depuis janvier-juin 1956, il nous semble important de produire un document multimédia qui rassemble à la fois les textes " historiques " de la fondation, les matériaux iconographiques et les enregistrements disponibles des témoignages des fondateurs aussi bien que des entretiens filmés avec les principaux acteurs de son histoire. Un tel document évolutif (écrit, sonore et audio-visuel) trouvera naturellement sa place sur le site ASSR.revues.org, et pourra s'enrichir au fur et à mesure de la quête des archives et des propositions de témoignages.

Dans une période d'accélération et de crise de l'aventure récente des éditions en sciences sociales, où les témoins de la première heure se dispersent, où les lieux d'inscription et les maisons d'édition se déplacent, où les revues se "dématérialisent » tout en multipliant leurs canaux d'édition et leur réseau de lecteurs, il n'est pas surprenant que la «commémoration » aille de pair avec un souci de «patrimonialisation ». Loin de nous cependant l'idée de transformer cette histoire exemplaire en un «lieu de mémoire » que l'on visiterait comme si nous

1. Rappelons entre autres, à ce sujet, la journée Gabriel Le Bras organisée le 6 avril 2006 par la Fondation Simone et Cino del Duca.

2. Voir en parallèle, le numéro de Social Compass, 51-1, 2004, p. 5-7, consacré au cinquantenaire de la revue, associant rétrospective et réflexion prospective sur les " cinquante ans à venir $»$. 
n'en étions plus. Ce qui frappe au contraire, à la relecture, c'est l'étonnante vivacité et proximité des enjeux et des questions qui se posaient alors et qui se posent encore dans toute entreprise d'étude scientifique des faits religieux ${ }^{3}$.

\section{D'une décennie à l'autre : quelques repères}

Le premier numéro des Archives de Sociologie des Religions date donc de janvier-juin 1956. La commémoration du dixième anniversaire, en 1966, fait état, comme il se doit, de " la nostalgie d'être en retard sur nos espoirs de 1956 ", mais déclare n'être " hantée par aucun aggiornamento » ${ }^{4}$. Le sous-titre de l'éditorial, signé H. D. ${ }^{5}$ : De la sociographie de la pratique religieuse à une pratique de la sociologie des religions, témoigne de la tension inaugurale et constitutive de l'entreprise. Le premier numéro n'était-il pas associé à la fois à la publication parallèle de la monographie paroissiale de J. Petit : «Structure sociale et vie religieuse d'une paroisse parisienne ", et à un mémorial dédié à Joachim Wach et à sa typologie des groupes religieux ? Dans l'avant-propos de Sociologie de la Religion (dont la traduction en français date justement de 1955), celui-ci évoquait le souhait de « voir se construire un pont au-dessus de l'abîme qui sépare, encore aujourd'hui l'étude de la religion et les sciences sociales, tâche dans laquelle ajoutait-il, l'anthropologie culturelle doit jouer un rôle important " ${ }^{6}$. Le devoir de mémoire emboîte le pas en poursuivant sur les deux fronts de l'empirie et de la théorie, de la classification et de la réflexion : d'un côté l'équipe produit ses premières tables signalétiques offrant un repérage de trois mille pages d'articles et cinq mille titres de thèses, ouvrages, recensés dans les vingt bulletins bibliographiques (les chiffres comptent) ; et de l'autre, est initialisé le principe de contributions régulières sur les grands auteurs de la sociologie allemande (Wach, Troeltsch, Weber, Simmel, etc.), puis sur les sociologues de langue française (Saint-Simon, Fourier, Comte, Durkheim, dans la perspective d'une collection. À noter que les grands auteurs qui dominent la théorie sociologique américaine du changement social (et religieux), à dominante "structuro-fonctionnaliste ", dans ces annéeslà (de Merton à Parsons) n'ont jamais eu droit à la galerie des ancêtres.

3. Ce texte ne m'appartient pas, sa dette par rapport aux nombreuses sources et témoignages utilisés et, à ce titre, largement cités, est évidente, mais l'extériorité du regard qu'il porte sur cette entreprise et l'orientation des questions retenues imposaient une signature. Qu'Émile Poulat, témoin irremplaçable de la première heure et historien averti de cette époque, soit particulièrement remercié pour ses précisions utiles et ses remarques bienveillantes.

4. ASR, 30, 1966, H. D. "D’une décennie à l'autre... ", p. 4.

5. Depuis sa rupture avec l'ordre dominicain en 1951, Henri-Charles Desroches signe Henri Desroche, H. D.

6. Joachim WACH, Sociologie de la Religion, Paris, Payot, 1955, trad. Maurice Lefèvre, Professeur à Dijon. 


\section{Quinze ans déjà}

Après la date de naissance de janvier-juin 1956, il est significatif que le groupe GSR, « bientôt identifié au groupe éditeur et rédacteur des Archives de Sociologie des Religions " ${ }^{7}$, se donne d'emblée une échéance de quinze ans, et donc de trente livraisons (deux numéros par an). Le bilan s'étale en fait de 1954 à 1969, la «quinzième année » étant une étape un peu mythique, un espace intervallaire qui prend son temps et avance sur plusieurs fronts. La partie principale est confiée à "l'archiviste » du groupe (É. Poulat) et se prolonge par d'autres bilans sur deux ou trois ans (1969, 1971 et 1972). L'enjeu et l'esprit de ce bilan restent ceux du rapport d'activité désormais classique d'un laboratoire du CNRS faisant le point sur l'ensemble des activités d'enseignement et de recherche du groupe.

Mai 68 est en arrière-fond, même si les «évènements » n'auront pas, apparemment, d'incidence sur l'équipe rédactionnelle. La période de l'après-68 est surtout marquée en 1970 par la mort de l'ancêtre fondateur, "le grand aîné qui tint à demeurer notre compagnon ", le Doyen Le Bras. L'heure est au bilan, pas vraiment à la commémoration, mais il y a de la re-fondation dans l'air... Les bulletins du périodique ne sont qu'un élément parmi d'autres de ce bilan. La collection des Archives sert de référence "totémique » pour témoigner d'une unité qui attend toujours d'être élucidée : "Et si une unité réelle se dégage de cette diversité spontanée c'est très certainement dans une lecture de la collection des Archives de sociologie des religions qu'un regard extérieur discernerait les éléments d'un consensus qui n'a pas encore éprouvé le besoin ou pris le temps de s'expliciter, et qui a maintenant passé quinze ans ${ }^{8}$.

1971, le no 31 revient sur le "Mémorial Le Bras ", confirme le «buissonnement » du groupe et le souhait de franchir une nouvelle étape en s'inventant un "nouveau totem » : un "Institut de Sociologie des Religions ", ou même "un Institut de Sciences Sociales des Religions », ISSR (on est en 1971...). Dans un esprit très proche de la pensée de $\mathrm{M}$. de Certeau, l'idée sous-jacente est que la meilleure manière de témoigner de sa fidélité à un fondateur est de procéder à une re-fondation : "ce ne serait pas trop d'une telle fondation pour perpétuer un tel fondateur...».

L'année 1973 est marquée par le changement de format et surtout de titre, et le ton de l'éditorial prend cette fois ouvertement des allures de refondation (de quoi retourner Le Bras dans sa tombe) : "En prenant le titre d'Archives de Sciences Sociales des Religions, qui la relie tant à la tradition marxiste qu'à celles issues de Weber ou de Durkheim, notre publication réaffirme son souci de rigueur théorique et son intérêt arrêté pour toutes les sciences de la pratique sociale. Globaliser - même de façon pluraliste - c'est comparer : comparer ne se

7. É. Poulat, ASR, 28, 1969, p. 10.

8. Idem, p. 12. 
peut que selon des critères élucidés. Sans renoncer à archiver des dossiers, ni au patient et rude labeur de la sociographie, ne pourrions-nous désormais accorder plus que nous l'avons fait jusqu'ici aux questions théoriques ? (p. 4, signé Le comité de rédaction). Mais cette science «multidimensionnelle» du fait religieux, attachée à ne pas séparer l'objet religieux des autres faits sociaux, pratiquant le comparatisme et la globalisation autant que la critique théorique, se veut en même temps fidèle à la tradition des Archives et, plus précisément, à cette dialectique de la continuité et de la discontinuité que H. Desroche a retenue de Bastide qui meurt justement en cette année 1973.

En 1976, et de fait en janvier 1977, les 20 ans ne sont pas un événement et encore moins un anniversaire significatif. Ils sont, selon le rédacteur en chef, toujours en place, un «moment de transit ». Le bilan n'est pas loin : "Rendezvous au bout d'une autre échéance non déterminée pour répertorier un nouveau stock de livraisons » (H. Desroche, p. 6). Et pourtant d'une couverture à l'autre, d'un titre à l'autre, et surtout en 1977 d'une périodicité à l'autre (trimestrielle) qui entraîne aussi une autre distribution entre la partie rédactionnelle, les notes critiques, les recensions bibliographiques, le paysage de la revue change. Après la disparition des recensions de thèses, il faudra néanmoins attendre 1992 pour voir l'abandon du Bulletin des périodiques.

Décembre 1980, les 25 ans, le $n^{\circ} 50$, tout un symbole, c'est «le temps des relèves " pour H. D., la « fin de l'épiscopat », selon son expression, le temps de passer la main après vingt-cinq ans de rédacteur en chef de la revue... Tous ses compagnons de la première heure reconnaissent que c'est lui qui fut le véritable initiateur et même "l'entrepreneur » de ce qu'il appelait «la firme Groupe + Archives de Sociologie des Religions " ${ }^{9}$.

Ces noces d'argent sont l'occasion d'un message de départ dans le style de Desroche : "Alter et idem : une requête d'Altérité dans une quête d'Identité ». Le ton du passage de relais entre la garde « descendante » et la garde « montante » est assez caustique... Que dire de l'opposition stigmatisée entre "leur imagination sédentaire et notre commémoration nomade»! Comme le note J. Séguy qui prend le relais : «Desroche était aussi une plume et un style très personnels, tout en bonds et rebonds, sautant d'une formule scolastique à une citation de Marx, d'une image biblique à quelque néologisme tout personnel... » ${ }^{10}$.

Pour quelqu'un qui aimait se définir comme un dissident de toute communauté et, par temps de crise, comme "le prototype de ceux qui sont sortis ", sa longévité à la tête de l'entreprise ne manque pas aujourd'hui d'étonner, surtout quand on réalise, comme le rappelle J. Séguy, que pendant plus de vingt ans le

9. Cité par R. Ducret, " Henri Desroche et le Centre Thomas More ", in Poulat et Ravelet, 1997, op. cit., p. 46.

10. In Memoriam, ASR, 87,1994 , p. 5. 
comité de rédaction des Archives s'est tenu dans les locaux mêmes du collège coopératif de l'avenue Franco-russe ${ }^{11}$.

C'est à partir du trentenaire (1987) que le devoir de mémoire et les obligations de la "commémoration" en tant que telle commencent à s'imposer à la rédaction : trente ans, déjà... Sur fond d'hommage à M. de Certeau, une grande figure présente/absente de la revue, la rédaction fait le choix significatif de la « liturgie silencieuse du labeur ordinaire continué »... C'est en fait l'occasion d'un grand numéro introduit par P. Ladrière et consacré à la réflexion sur les sciences sociales des religions ${ }^{12}$. La convocation de grands noms, Vernant, Arkoun, Bourdieu, Langlois, Deconchy, Vincent, etc., est précédée de la publication d'un article, à la fois bilan et "manifeste ", de Danièle Hervieu-Léger : "Faut-il définir la religion?", qui prend une fois de plus l'accent d'une refondation continuée :

"La "célébration" de l'anniversaire des Archives, nous dit DHL, ne peut que s'inscrire dans ce mouvement de retour sur soi dont on attend qu'il dessine des orientations pour l'avenir. Après tout, l'aventure de la revue n'est-elle pas la trajectoire des sciences sociales des religions en France, depuis trente ans ? Dire cela ne signifie pas que celles-ci aient jamais prétendu embrasser et récapituler le champ entier de la discipline : la sociologie des religions, fort heureusement, a toujours débordé les limites d'une revue spécialisée, en même temps que celle du groupe qui en avait été l'initiateur! Mais l'ambition de publier des travaux originaux et de stimuler la recherche en favorisant échanges et débats, n'y fut jamais séparée du souci de se faire l'écho aussi fidèle et complet que possible de l'évolution et des productions de la spécialité ${ }^{13}$.

L'héritage toujours vivant qu'il s'agit alors de perpétuer est donc bien celui d'une « revue-laboratoire " animée par un groupe de type coopératif rassemblant les principaux chercheurs du champ, et se retrouvant dans la matrice d'accueil de la «sociologie des religions».

Enfin, les «quarante ans déjà ..." (1996) conduisent la nouvelle rédactrice en chef Danièle Hervieu-Léger à confier « l'œuvre de mémoire » à F.A. Isambert : «La meilleure façon de célébrer l'événement est, selon nous, de faire retour sur la trajectoire de la revue, et de réfléchir, sous la conduite de l'un de ceux qui ont le plus contribué à lui donner l'audience qui est la sienne, à un parcours intellectuel qu'il importe aujourd'hui de poursuivre sans cesser d'innover ». Cet article remarquable est, depuis le bilan à quinze ans de É. Poulat, une des biographies des figures intellectuelles de la revue les plus intéressantes qui soient à ce jour.

11. J. SÉGuY, "Communauté et Religion », in É. Poulat et C. Ravelet, 1997, op. cit., p. 23

12. ASSR, 63-1, janvier-mars 1987. Les textes sont issus de la session annuelle de l'AFSR (Association Française de Sociologie Religieuse).

13. ASSR, 63-1, p. 12. 


\title{
In Memoriam ...
}

Le devoir de mémoire s'exerce naturellement aussi dans les In Memoriam qui ponctuent l'histoire de la revue, et même qui l'inaugurent si l'on retient le mémorial initial consacré à Joachim Wach dès le numéro 1 . Certains relèvent de l'hommage incontournable aux ancêtres et aux pères de la revue, et témoignent de filiations connues :

Duveau G. 1903-1958 (G. Le Bras)

Léonard E.G. 1891-1961 (G. Le Bras)

Le Bras G. 1891-1970 (H. Desroche)

Desroche H. 1914-1994 (J. Séguy)

Mehl R. 1912-1997 (J.-P. Willaime)

Mais d'autres hommages témoignent de parentés plus souterraines ou de parrainages collatéraux :

\author{
Griaule, 1898-1957 (G. Dieterlen) \\ Gernet, 1882-1962 (J.-P. Vernant) \\ Métraux, 1902-1963 (R. Bastide) \\ Massignon, 1883-1962 (V. Monteil) \\ Bastide, 1898-1974 (H. Desroche)
}

M. de Certeau, mort en janvier 1986, occupe la place de l' " Absent » dans l'histoire des in mémoriam. La publication posthume d'une communication antérieure était prévue dans le numéro du trentenaire de 1987, mais « sa disparition a rendu impossible la publication de son texte ».

\section{Retour sur l'histoire paradoxale d'une revue}

\section{Naissance d'une revue, vie et mort du Bulletin Bibliographique}

La publication par le Groupe de Sociologie des Religions du CNRS au premier semestre 1956 du premier numéro des Archives ne correspond pas vraiment à un projet éditorial de "revue " au sens où on l'entend aujourd'hui, même si H. D. avait déjà l'expérience d'Économie et Humanisme. C'est un projet de travail de recensement systématique des ressources bibliographiques concernant l'étude des faits religieux qui n'est pas sans rapport avec l'esprit de documentation exhaustive de l'Année Sociologique pratiqué par Mauss (qui comportait déjà un volet Sociologie religieuse). L'entreprise de rassemblement et d'ordonnancement systématique des œuvres dispersées du Doyen Le Bras, initiée par Henri Desroche, aidé de Juliette Turlan, dans l'année 1953-1954, a donné l’impulsion initiale ${ }^{14}$. La commande de bibliographie de l'Unesco, le fameux Trend

14. Sur cette période voir, évidemment, É. Poulat, ASR, 28, 1969, p. 6-7. 
Report de 1954-1955, a conforté au sein de l'équipe une sorte d'habitus scientifique du travail sur fiches, du tableau à double entrée et de la matrice carrée, susceptible de s'appliquer à toutes les données ${ }^{15}$.

Les « archives » désignent moins, au départ, une " revue » qu'un " bulletin ", un "périodique ", ou tout simplement des "notes " et des " fiches ", au service d'un projet scientifique de catégorisation, de classement et de typologie de la matière disponible. Il s'agit d'un instrument au service d'un projet scientifique supporté par un groupe de recherche qui n'a pas d'autre structure d'organisation que la revue : le comité de rédaction des Archives est de fait le comité de direction du groupe.

Ce qu'on appelle la "partie rédactionnelle » est, au niveau des entrées, plutôt restreinte et le restera pendant plus de quinze ans: les tables signalétiques (ce recensement des recensements) font état de $4 \%$ des entrées proprement rédactionnelles de 1956 à 1970 (un pourcentage d'entrées signalétiques qui ne préjugent pas néanmoins de la place occupée). Il faudra attendre pour voir se dessiner de vrais numéros thématiques sur "Religion et Développement ", "Religion et Socialisme », «Religion et changement social », sur les «sectes ", " les messianismes ", la "Religion populaire ", ou sur les aires culturelles : "Les religions en Asie du Sud Est ", "Sociologie des religions au Brésil » ou autres. Plusieurs rubriques cohabitent en fait et se poursuivent parallèlement dans un même numéro ou rebondissent d'un numéro à l'autre: sociographie des pratiques (catholiques, protestantes, juives); études d'auteurs connus ou méconnus; état des lieux de la sociologie dans les divers pays; réflexion épistémologique ou méthodologique sur les sciences sociales du religieux et les sciences religieuses ou la théologie. La revue se fait l'écho régulièrement des thèmes de conférences internationales ou de colloques (L'image de la mort, etc.).

Après l'abandon du Bulletin des Thèses (faisant désormais double emploi par rapport à certains services documentaires), puis, à partir du $\mathrm{n}^{\circ} 33$, le renoncement aux résumés d'articles offerts par le Bulletin des Périodiques qui crée une sorte d'inflation signalétique de ce dernier, c'est toute l'entreprise de prospection et surtout de classement bibliographique qui est ébranlée. Le bilan des « 7000 fiches en 15 ans » s'interroge, au moins au regard des tables signalétiques, sur l'utilité de ce «travail ingrat et astreignant ${ }^{16}$. Les secondes tables signalétiques (1971-1976) témoignent d'un «certain sentiment de frustration, voire d'amertume ", face à l'échec douloureux de l'élaboration d'un index matières, dernier

15. En quittant la revue en 1980, H. Desroche se replie entre autres sur les Archives de Sciences Sociales de la coopération et du développement, et le projet de recensement de tous les écrits de Bastide qu'il poursuit plus tard avec C. Ravelet, dans le cadre de Bastidiana, autant que la création de la revue autobiographique d'Anamnèses (1990), témoignent encore de cet habitus des archives.

16. ASR, 1972, p. 7. 
soubresaut de l'entreprise typologique. Le renoncement au Bulletin des périodiques en $19922^{17}$, et le resserrement du B.B. - un Bulletin bibliographique des ouvrages qui ne répond plus à un projet de recensement exhaustif et encore moins de classification thématique - consacre la fin d'une époque.

Le premier paradoxe est donc que l'épuisement progressif de cette entreprise de recensement exhaustif de thèses, d'articles et d'ouvrages, et l'échec de la grande ambition de typologie des faits religieux qui lui donnait sens ${ }^{18}$, a engendré, au fil du temps, une revue dont "la partie rédactionnelle » n'a cessé de s'étoffer et de se construire.

\section{De la « sociologie religieuse » à la « sociologie des religions »}

Le consensus affiché de la communauté renforcée est réitéré, à chaque étape de transition, par la commémoration de la continuité. Le miracle de cette fidélité continuée à une entreprise scientifique et éditoriale reste entier au regard des divergences d'engagement scientifique, religieux et politique des membres de l'équipe. Comme le rappelle J. Séguy dans son In Memoriam à H. Desroche, pour ce dernier, comme pour toute l'équipe : «le sociologue devait non seulement chercher, réfléchir, parler et écrire, mais il devait aussi “faire”. Que de débats et de discussions sur ces problèmes et entre nous, dans les années 60, et au-delà encore ... La sociologie pouvait-elle s'engager, et si oui, jusqu'à quel point ${ }^{19}$. On sait que pour H. Desroche l'investissement dans les entreprises coopératives est le prolongement naturel de son engagement d'intellectuel organique du catholicisme de gauche ${ }^{20}$ et la contrepartie de son retrait du monde religieux.

Entre le doyen Le Bras, catholique bretonnant traditionnel, familier de Dieu et du diable, "prince du droit canonique », proche de la hiérarchie catholique

17. ASSR, 77, Danièle Hervieu-LÉGER, « Les Archives : des changements, pour la continuité d'un projet ». Les raisons résumées par J.-P. Deconchy ne sont pas seulement matérielles et techniques, elles reposent une fois de plus le problème des fondements du tri, au niveau des articles dispersés dans toutes sortes de revues de plus en plus nombreuses, de ce qui relèvent $\mathrm{du}$ « religieux» ou pas.

18. Quarante ans plus tard, l'auteur du texte-programme du $\mathrm{n}^{\circ} 1$ : « Pour une bibliographie systématique en sociologie des religions ", tire un bilan très lucide : "Notre but était de mettre en place une structure qui se prolongerait dans les Archives par une bibliographie systématique continue. Le temps nous manqua et nous remîmes le projet pour le moment où nous aurions pu élaborer un plan bibliographique véritablement scientifique. Ce temps faillit venir mais échoua à arriver. " ASSR, 93, op. cit., p. 16.

19. ASSR, 87, 1994, p. 5.

20. Voir sur ce sujet D. Pelletier, «Signification du Marxisme (1949), Histoire d'un livre ", p. 154, in É. Poulat et C. Ravelet, 1997, op. cit., p. 154. On ne peut qu'espérer qu'un travail aussi remarquable que celui de Denis Pelletier sur l'histoire d'Économie et Humanisme (Cerf, $1996)$ soit effectué un jour à propos de la «firme » GSR-ASSR. 
et de Rome, et même «conseiller pour les affaires religieuses » (CAR) du ministère des Affaires étrangères - pas très à l'aise, au-delà des relations d'amitié personnelle, vis-à-vis de l'entreprise de ce groupe de « sociologues » inspirés par le marxisme -, un ex-dominicain Charles-Henri Desroches, en rupture d'Église et en quête de communauté utopique et d'esprit coopératif à la Fourier, et un Émile Poulat, historien critique de l'Église, réveillant les enjeux de la crise moderniste, et soulevant sérieusement la question explosive des prêtres-ouvriers, l'entente n'était pas si évidente. On peut se demander comment ces trois «mousquetaires» qui forment avec F. Isambert et J. Maître, les «cinq doigts de la main » - tous des hommes d'engagement - ont pu se retrouver sur un projet scientifique de sociologie objective, positive et descriptive des "faits religieux ", à distance des partis pris idéologiques et confessionnels ?

Le second paradoxe est que l'entreprise scientifique fondatrice de ces "sociologues des religions » du CNRS ait pu être «patronnée » (et cautionnée) par un maitre qui n'en était pas vraiment, qui se méfiait " des initiateurs et fondateurs " et préférait les «mainteneurs ${ }^{21}$. Ses disciples ne cesseront de faire comprendre que le maître ne partageait pas l'enthousiasme des convictions et des espérances de cette aventure scientifique. Ne parlons pas du patronage protestant du maître G. Léonard, le croyant réformé, grand historien du protestantisme et directeur d'études, qui selon les explications mêmes de G. Le Bras, n'appréciait guère les méthodes sociographiques et encore moins les typologies sociologiques, et surtout craignait avant tout dans cette entreprise la tentation de "réduction du divin à l'humain " (In memoriam, G. Le Bras, 1961).

De la «sociologie religieuse » des enquêtes de pratiques chère à G. Le Bras, plus ou moins héritée de l'école durkheimienne et plus particulièrement de Mauss ${ }^{22}$, à la "sociologie des religions " de ces intellectuels progressistes chrétiens, lecteurs de Marx, Engels, Weber, et Durkheim, inspirés par l'entreprise de typologie des groupes religieux de Joachim Wach, le saut est important. Comme en témoigne, H. Desroche : "G. Le Bras n'était pas sans être chiffonné par le label "sociologie des religions" substitué à celui de "sociologie religieuse", substitution en laquelle il repérait, à juste titre, l'emblème d'une différenciation et les risques d'une aventure ${ }^{23}$. Entre une science empirique de recensement des

21. É. Poulat, L'ère postchrétienne, Paris, Flammarion, 1994, p. 64.

22. Dans un hommage à l'Année Sociologique (ASR, 21, 1966, p. 53), G. LE Bras déclarait ainsi sa dette: "Me sera-t-il permis de reconnaître ma dette envers Marcel Mauss ? ... Dès 1907, analysant l'ouvrage d'Arréat, Le sentiment religieux en France, Mauss avait recommandé l'observation de toutes les manifestations de la vie religieuse, et avec force, les statistiques. En 1925, il précisait sa pensée en des termes qui nous ont beaucoup frappé. "La force d'une Église se mesure au nombre et à la richesse de ses temples, au nombre de ses croyants et à la grandeur de leurs sacrifices (...). Manié avec prudence et intelligence, le procédé statistique est non seulement le moyen de mesurer mais le moyen d'analyser tout fait social...” (A.S. N.S. II, p. 152) ", p. 53 .

23. ASR, 29, 1970, H. Desroche, In Memoriam, p. 4. 
données, résistante à la comparaison et à des généralisations hâtives, interpellant les fondements des Églises établies, et une science faisant confiance à l'imagination théorique et à l'excitation des typologies, l'incompréhension se creuse avec les années.

Le clivage interne au groupe de sociologie des religions ne passe d'ailleurs pas tant par l'opposition entre empiristes et théoriciens, que par le penchant entre deux voies possibles de la synthèse. Comme le fait remarquer très subtilement F. Isambert à propos de l'élaboration du travail bibliographique systématique, pris dans la tension entre recensement et classement : "Aussi bien les synthèses les plus valables se sont-elles présentées sous forme comparative, soit historique, soit typologique (voir la sociologie de la religion de J. Wach ou la phénoménologie de la religion de Van der Leeuw)... » ${ }^{24}$. Entre la gratuité des théories et des typologies a priori qui brassent trop larges, et les illusions d'une théorie inédite qui surgirait de l'accumulation des faits et de la simple «manipulation des paquets de fiches ", le débat est ouvert et lucide. É. Poulat confirme que H. Desroche, comme l'illustre son goût des tableaux ou matrices des mouvements messianiques ou des modèles de coopération, "préférait la typologie combinatoire aux conceptualisations généralisantes $» 25$.

Dans son entretien avec H. Desroche, en 1970, G. Le Bras se refuse néanmoins (en déjouant le piège de la question) à entendre parler de rupture entre la sociologie des religions des grands classiques (Durkheim, Weber) et une sociologie religieuse, d'esprit à la fois empirique et pastorale, qu'il aurait incarné ${ }^{26}$. Pour faire équilibre aux chantiers bibliographiques et sociographiques qui mobilisent le groupe de sociologie des religions dans les premiers temps, la revue ne cessera dès ses premiers numéros de consacrer une large place aux grands auteurs de la tradition sociologique. Le principe même de contributions tournantes sur les auteurs de référence rythme la vie de la revue : Troeltsch, Simmel, Bonhöffer, et Weber, Comte, Durkheim, etc. On retrouve là le souci explicite de ne pas séparer l'entreprise empirique et le goût de l'enquête de l'inspiration des grands ancêtres attachés aux grandes théories ${ }^{27}$. J. Maître nous rappelle comment " sans aucun ostracisme envers les travaux développés outre-Atlantique, la sociologie des religions s'est fondée avec détermination sur les classiques européens, à

24. F. IsAmbert, "Pour une bibliographie systématique en sociologie des religions », ASR, 1, 1956, p. 142.

25. É. Poulat, "Henri Desroche, compagnon et maitre ", in É. Poulat et C. Ravelet, Henri Desroche: un passeur de frontières, hommage, Paris, L'Harmattan, 1997, p. 10.

26. H. Desroche et G. Le Bras, "Religion Légale et Religion Vécue ", Entretien avec Gabriel Le Bras, France-Culture, février-mars 1968, ASR, 29, 1970, p. 18.

27. Il faut peut-être, à ce sujet, nuancer quelque peu le propos de D. Hervieu-Léger sur la "réserve théorique » surprenante du Groupe de sociologie des religions à la française, comparé à leurs collègues sociologues anglo-saxons, Cf. La religion pour mémoire, p. 20. 
commencer par Marx et Engels, grâce à la vigueur de la lecture qu'avait su en faire Henri Desroche ${ }^{28}$.

Il faut dire que ces jeunes "sociologues des religions ", invités par le maître à s'ouvrir aux religions du monde (et à la question, pour lui mystérieuse, de l'altérité radicale, celle de l'irréligion... pour ne pas parler de l'athéisme), se précipitent sur les non-conformismes et sur les marges du religieux autant que sur toutes "les religions de contrebande ", pour reprendre le titre d'un ouvrage de H. Desroche. Les sujets de thèses et les œuvres des uns et des autres en témoignent : des Shakers américains aux assemblées anabaptistes-mennonites de France. Les premiers numéros sont dominés par des contributions sur les messianismes et les prophétismes (Desroche), sur les sectes millénaristes et les dissidences religieuses (Séguy), et les spécialistes des aires culturelles (Brésil, Océanie, Afrique, Asie) sont largement mis à contribution. Comme le dit Le Bras, en toute lucidité (cité par É. Poulat ${ }^{29}$ ) : “À moi l'Église, à vous les sectes ». On peut dire que dès le départ la tension entre le religieux institué et le religieux instituant, entre la religion et le religieux hors religion, est constitutive du champ des préoccupations de la revue, et qu'on la retrouve aujourd'hui sous d'autres formes.

La nécessité, constamment rappelée, de la séparation entre une science des religions, dominée par le modèle universitaire de la scientificité sociologique, et une sociologie appliquée, sociologie confessionnelle ou pastorale, sociologie d'expertise et de conseil de "pasteur sociologue » ou de sociologue pasteur, n'allait pas de soi. On a du mal à imaginer, aujourd'hui, qu'une entreprise scientifique cautionnée par le CNRS ait pu subir quelque pression, même indirecte, des autorités ecclésiastiques, dans une France "laïque ", mais la simple comparaison avec d'autres entreprises similaires voisines est éclairante. La longue histoire de la dépendance de la revue Social Compass par rapport à l'institution catholique universitaire et la confusion entretenue entre sociologie des religions, doctrine sociale et pastorale catholique, se retrouvent sans ambiguité dans les termes de la présentation éditoriale de la revue réaffirmée en 1960 : "Le but de cette revue catholique consacrée aux études socioreligieuses est double : faciliter l'utilisation des méthodes sociologiques dans la pensée et l'action des chrétiens et apporter en sociologie la contribution des catholiques dans un domaine qui leur est propre, la sociologie de l'Église catholique ${ }^{30}$. É. Poulat a également

28. J. MAÎTRE, « Henri Desroche, introducteur du marxisme dans le champ de la sociologie des religions en France ", in É. Poulat et C. Ravelet, 1997, op. cit., p. 165. J. Maître note néanmoins, non sans ironie, que malgré la continuité profonde qui existait pour H. D. au sein des préoccupations de la sociologie allemande, ni Marx ni surtout Engels, n'ont eu droit au sommaire des grands auteurs de la revue.

29. É. Poulat, L'ère postchrétienne, op. cit., p. 48.

30. Cité par Manuel J. MejIDO, "On the Genesis and Transformations of Social compass", Social Compass, 51-1, 2004, p. 31. Voir également le témoignage de F. HoutArd, « Les 50 ans de Social Compass : autobiographie d'une revue qui s'est trompée de nom », idem. 
rappelé comment la CISR (Conférence Internationale de Sociologie Religieuse), conçue et fondée au départ, au moins dans l'esprit de J. Leclerq, en 1948, comme une organisation scientifique non dénominationnelle, s'est trouvée dès 1951 remise dans le droit chemin et re-confessionnalisée, avec la complicité d'une base formée autant de sociologues catholiques que de catholiques sociologues ${ }^{31}$. Il faudra attendre 1971 pour qu'un mouvement consensuel rompe définitivement le cordon ombilical avec la matrice confessionnelle.

Le principe de la complémentarité des intérêts scientifiques et religieux est de fait très discutable, et discutée. Dans ses «Réflexions sur les différences entre sociologie scientifique et sociologie pastorale " ${ }^{32}$, G. Le Bras rappelle le principe de base d'une science commune opérant par "paliers ", de la description des faits jusqu'à l'explication des causes en passant par la comparaison des structures de la vie des groupes, la sociologie des religions représentant le plus haut «degré » de l'entreprise. L'indépendance postulée des paliers permettrait en quelque sorte de sortir à chaque étape ou à chaque étage, le premier étage étant généralement suffisant pour le pasteur, les étages plus élevés comportant des risques pour la foi : "sa profession de foi lui interdit de réduire le sacré au profane » ou exclut « une explication naturelle du surnaturel ${ }^{33}$. Il est clair que cette construction à étage n'est qu'un artifice ${ }^{34}$. En précisant lui-même que le pasteur est invité à "borner la typologie aux exigences du ministère » ${ }^{35}$, G. Le Bras admet d'ailleurs lui-même que pour une sociologie pastorale qui entend s'assurer le double profit de la scientificité de l'entendement et de l'efficacité de l'action, la description est logiquement commandée par des catégories de classement et des typologies de pratiquants (et de non pratiquants) qui font sens pour le ministère.

\section{Un « œcuménisme » interconfessionnel, interdisciplinaire et international ?}

On peut, à ce propos, s'interroger sur la gestion, dans la longue durée, des ancrages ou des héritages confessionnels au sein même de la revue ? Dans les préalables du numéro 1 de 1956, il est bien dit : « Des hommes de toutes croyances et incroyances voisinent dans le cortège et nous n'avons point à connaître leurs sentiments. Cette revue ne saurait être au service d'aucune doctrine, confessionnelle ou anti-confessionnelle ${ }^{36}$. La profession de foi en la neutralité de la

31. É. Poulat, «La CISR, de la fondation à la mutation : réflexions sur une trajectoire et ses enjeux ", in Social Compass 37-1, 1990, p. 11-33. Il n'est pas inutile de rappeler le destin parallèle de l'histoire de l'AFSR qui émane du Centre catholique de sociologie religieuse.

32. G. LE BRAS, "Réflexions sur les différences entre sociologie scientifique et sociologie pastorale ", $A S R, 8,1959$, p. 5-14.

33. Idem, p. 12.

34. Cf. É. Poulat, L’ère post-chrétienne, op. cit., p. 52-53.

35. G. LE BRAS, op. cit., p. 12.

36. ASR, 1, 1956, p. 6. 
science, de la part de chercheurs du CNRS, est on ne peut plus explicite, mais on serait à vrai dire bien en peine de situer des «incroyants ", et encore plus des "athées ", dans le cortège. Ceux que H. Desroche, dans son œcuménisme à bras ouvert, appelle "des ressortissants d'une non-religion nourrissant pour la ou les religions tantôt une affinité, tantôt une agressivité, tantôt une indifférence $"{ }^{37}$ ne sont pas exclus, mais ils sont ailleurs. La distance prise par rapport à l'institution, le privilège accordé au non-conformisme religieux, la fascination pour l'irréligion, ne conduisent pas pour autant, comme le confirme à sa façon l'intérêt pour une "sociologie religieuse de l'irréligion ", à comprendre et encore moins à mettre en pratique une épistémologie scientifique fondée sur une posture d'indifférence pure et simple par rapport au religieux, ou une absence d'intérêt pour les choses religieuses, au sens de Bourdieu.

Le pluralisme religieux, reflété par l'étendue des spécialités des rédacteurs et des contributeurs, fait son chemin, lentement: le «sociologue catholique du catholicisme " G. Le Bras, s'est adjoint très tôt la collaboration de ce «croyant réformé » de tendance évangélique, grand historien du protestantisme, É.-G. Léonard ${ }^{38}$, lui-même relayé plus tard par R. Mehl, philosophe et théologien protestant converti à la sociologie du protestantisme ${ }^{39}$; les études consacrées au judaïsme, cette "ethno-religion ${ }^{40}$, ont aussi leur filiation (D. Bensimon) D'autres passages de relais sont moins évidents, comme sur l'islam (V. Monteil, J.-L. Triaud, C. Hamès), ou les autres religions non chrétiennes de "l'œcumène " : du boudhisme ou du chamanisme aux religions africaines ou afro-brésiliennes. Il faudrait reprendre, par le biais des relations de maitre à disciple, des jurys de thèse, des hommages et "in memoriam ", l'arbre généalogique des filiations internes des spécialistes des confessions, sa diversification, ses logiques souterraines et ses ruptures ${ }^{41}$. La confessionnalité n'est pas, si l'on peut dire, un péché, elle pose avant tout une question épistémologique : celle des relations que les diverses traditions religieuses entretiennent avec les sciences sociales, et réciproquement. Le problème ne concerne d'ailleurs pas seulement le sociologue du catholicisme ou du protestantisme, ou le spécialiste des études juives, les anthropologues des religions "africaines » ont aussi à interroger leur "intérêt " par rapport au "sacré sauvage » (R. Bastide) ou au " génie du paganisme » (M. Augé).

H. Desroche évoquait régulièrement le principe déontologique d'un œcuménisme scientifique ou «méthodologique » inhérent à l'étude de la pluralité des

37. H. DeSroche, "CEcuménismes, Sciences de l'Homme et Créativités ", ASSR, no 44-2, 1977, p. 153-173. p. 3-5.

38. Gabriel Le Bras, «In Memoriam Émile-G. Léonard (1891-1961) », ASR, 14, 1962,

39. J.-P. Willaime, «In Memoriam Roger Mehl (1912-1997) », ASSR, 98, 1997, p. 5-7.

40. Cf. le rapport à quinze ans, $A S R, 28, \mathrm{p} .40-41$.

41. Comme le soutient M.J. Mejido : "The history of Social Compass is testimony to the fact that denominational distinctions have been epistemologically relevant in the development of the sociology of religion ", Social Compass, 51-1, 2004, p. 28. 
religions et à leurs « divisions » ${ }^{42}$, associé au souci des religions de " l'œcumène » ${ }^{43}$, ou même ouvert aux «non-religions » porteuses de convictions globales et de visions du monde universelles: "Il est difficile à un sociologue des religions, qu'il soit lui-même religieux ou non, surtout s'il est quelque peu anthropologue ou historien, de trouver son aboutissement, sinon dans une sociologie œcuméniquement polarisée ${ }^{44}$.

Cet œcuménisme méthodologique n'est sans doute pas à confondre avec l'engagement dans un œcuménisme théologique ou religieux (qui était déjà dans l'air du temps), mais la traduction de l'un dans l'autre n'était pas exclue. Dans l'anniversaire des dix ans de la revue, $\mathrm{H}$. Desroche rappelle le pari, qui tient toujours mais n'est pas encore gagné, sur « une œecuménologie inter-confessionnelle, inter-nationale, inter-idéologique et inter-disciplinaire " ${ }^{45}$. L'œcumène, c'est le véritable mana de la sociologie religieuse de Desroche, et il n'est pas surprenant, comme le rappelle très bien J. Séguy ${ }^{46}$, que l'aboutissement de cette quête de l'œcumène, prolongement de l'agapè, se trouve en définitive sur le terrain d'une pratique coopérative rassemblant des compagnons de toutes convictions au service d'un développement humain communautaire.

L'autre défi que la revue s'est efforcé de relever est enfin celui de la pluridisciplinarité ou de l'interdisciplinarité justifiée par la communauté d'objet: le fait religieux. Sous l'appellation « sciences sociales " (plus ponctuellement « sciences humaines »), qui précède son adoption comme titre de la revue en 1973, se développe un souci constant d'ouverture disciplinaire au départ fortement marquée néanmoins par la matrice de la scientificité sociologique. Les revues sœurs des Archives sont naturellement, en dehors de la revue mère de L'Année Sociologique, les Cahiers Internationaux de Sociologie et la Revue Française de Sociologie. Le débat fondateur ou initiatique sur la place de la théologie (les fameuses " traditions théologiques »), ou les rapports à la philosophie, constituait un préalable, mais ces questions sont mises sous le boisseau avec le temps. Si certaines disciplines ont leur place naturelle: sociologie, ethnologie (plus qu'anthropologie), et histoire, d'autres comme la psychologie (historique, sociale, ou freudienne) ou la psychosociologie, l'économie, ou même le droit, ont une place plus oscillante, marginale ou éphémère, souvent liée à la spécialité ou aux explorations de tel ou tel membre fondateur poursuivant son sillon (le droit canon de G. Le Bras, la psychologie sociale de J.-P. Deconchy ${ }^{47}$, etc.). Au départ, le triangle est plutôt sociologie, psychologie, histoire (selon Ladrière, $c f . \mathrm{n}^{\circ}$ du trentenaire),

42. Cf. l'entretien de 1970 avec G. Le Bras, op. cit., p. 19.

43. ASR, 31, 1971, p. 4.

44. H. DesRoche, "Ecuménismes, Sciences de l'Homme et Créativités ", ASSR, op. cit.

45. H. Desroche, $A S R, 30,1966$, p. 3.

46. J. SÉGUY, "Communautés et religion ", op. cit., p. 31.

47. Cf. J.-P. Deconchy, "Les méthodes en psychologie de la religion », ASSR, 63-1, 1987, p. 31-83. 
mais avec le temps la psychologie s'efface, et, par contre, l'ouverture sur les «aires culturelles " (Afrique, Brésil, Inde), ou les terrains anthropologiques, se développent, entre autres par le biais des messianismes, syncrétismes et millénarismes en tout genre.

\section{Aujourd'hui...}

La revue des Archives est désormais publiée par les Éditions de l'EHESS et non plus par le CNRS. Le "Groupe de Sociologie des Religions » du CNRS a essaimé et s'est dispersé dans divers établissements (EHESS, EPHE, Université) et plusieurs organismes de recherche. Ce n'est plus une équipe de recherche restreinte qui trouve dans la revue sa vitalité scientifique et son identité de référence, mais un concours de centres et de laboratoires plus ou moins représentés dans un comité de rédaction renouvelé et élargi. Le comité de lecture (lecteurs et recenseurs) constitue plus que jamais un réseau virtuel et plus ou moins anonyme de bénévoles dont la disponibilité et l'efficacité restent étonnantes.

La revue a failli, dans les changements en cascade qui ont marqué sa nouvelle inscription et décidé de son nouveau look, changer aussi de titre et cesser, à l'approche du cinquantenaire, d'être tout simplement les Archives pour devenir quelque Revue des Sciences Sociales des Religions. Mais à la réflexion, l'ère de l'édition électronique et le souci du " patrimonial » ont redonné vie à cet emblème totémique et enrichi ses résonances analogiques. Autre symbole, le «B.B. », le Bulletin bibliographique, aurait pu disparaitre dans son édition "papier ", la mise en ligne des recensions et notes bibliographiques sur le site ASSR.revues.org assurant aux auteurs et aux recenseurs, et surtout aux ouvrages, une diffusion rapide. Mais là encore, l'attachement de nos abonnés (et même des auteurs et recenseurs) à l'édition papier du Bulletin nous fait un devoir de conserver cette institution à part entière. Les grandes ambitions de recensement systématique et surtout de classement thématique de la littérature en sciences sociales des religions font partie désormais du passé, mais pour nous qui sommes de grands voyageurs (et encore plus pour les sédentaires) le parcours suivi ou aléatoire de ces comptes rendus inégaux, des ouvrages les plus proches aux plus lointains, reste un rendez-vous inestimable et incomparable.

Que dire aujourd'hui du projet scientifique et éditorial d'une revue qui postule, non l'irréductibilité, mais au moins la spécificité de l'étude des faits religieux, soumise au prisme de chaque discipline concernée (histoire, sociologie, anthropologie) ? Le problème n'est pas nouveau. M. de Certeau, récusant l'idée de "sciences religieuses ", était convaincu pour sa part (la leçon de Lévi-Strauss n'étant pas très loin) de l'impossibilité de définir des critères du religieux, et cela non seulement du fait de l'élasticité du phénomène dit religieux et de l'indiscernabilité des frontières mais surtout du fait de la situation épistémologique des sciences humaines qui lie la manière de comprendre les pratiques et les objets au renoncement à toute régionalisation des faits religieux et même à l'exclusion 
de toute spécificité du «matériau » religieux ${ }^{48}$. H. Desroche avouait lui-même, quelque temps avant de passer la main : " Je me sens de plus en plus mal à l'aise de devoir traiter les phénomènes religieux comme des phénomènes à spécificité tranchante. Je voix bien qu'ils ont présentement une différence spécifique, mais dans un triple genre commun : celui d'une forme de conviction globale, celui d'une créativité culturelle, celui enfin d'une expérience du sacré » ${ }^{49}$.

Sur le fond, les grands débats de l'âge « métaphysique » sur l'essence de la religion, que les fondateurs des Archives ont tacitement contournés, font pourtant retour dans les préoccupations philosophiques d'aujourd'hui, comme en témoigne l'engouement de nos philosophes ou penseurs pour le religieux. Sur le terrain des sciences sociales, les discussions importantes sur la recherche "positive " d'une définition substantielle du religieux (le sacré et ses avatars), dans les formes éclatées ou disséminées qu'il peut prendre en régime de modernité (ou de surmodernité), ou le souci légitime de la recherche de repères méthodologiques minimaux permettant de cerner ce qu'est un "fait religieux », en présence du religieux ordinaire ou de religiosités séculières, restent à ce jour totalement ouverts, et il faut s'en réjouir... La relativité et la réflexivité sont au principe même de ces entreprises récurrentes et stimulantes, par définition soumises à révision et discussion, à l'épreuve des faits, leur portée heuristique étant totalement incompatible avec l'intention de fixer quelque frontière du religieux.

Parallèlement, la matrice sociologique qui servait d'accueil aux diverses sciences sociales du religieux a quelque peu implosé : dans les centres de recherche qui relèvent, en France, des études sur les religions, on trouve désormais plus d'historiens, d'anthropologues ou de politologues, que de sociologues "proprement dits ». La pertinence de l'objet religion ne concerne pas seulement le sociologue de nos sociétés confronté au déclin ou à la dissémination du religieux, mais tout autant l'anthropologue ou l'historien s'efforçant de penser la complexité d'autres régimes du religieux ou d'autres formes de religiosité. L'approche pluridisciplinaire reste sur ce plan le meilleur garde-fou contre les tentations essentialistes ou les dérives ethnocentristes. Comme le souligne D. Hervieu-Léger, en connaissance de cause: "Dans la tension dynamique qui les arc-boutent les unes aux autres, les différentes approches enserrent l'objet dans un réseau définitionnel, qui permet précisément de dépasser le projet d'accéder, en une ultime et exclusive définition, à l'essence même de la religion ${ }^{50}$.

48. M. de Certeau, La faiblesse de croire, Paris, Seuil, 1987, p. 194-196.

49. H. Desroche, "Ecuménismes, Sciences de l'Homme et Créativités ", ASSR, 44-2, 1977, op. cit.

50. D. Hervieu-LÉger, «La religion, mode de croire », in Revue du MAUSS, 22, 2003, p. 147. Y. Lambert, dans une mise au point très claire conclut dans le même sens : "l'intérêt d'une définition est également relatif à un type de questionnement. Il ne saurait donc y avoir de définition qu'au pluriel (au-delà de traits minimaux), et de frontière qu'en continuum (audelà de territoires reconnus) ", Y. LAMBERT, "La "Tour de Babel” des définitions de la religion ", Social Compass, 38-1, 1991, p. 82. 